\title{
Microfluidic Rheology of Non-Newtonian Liquids
}

\author{
Salvatore Girardo, Roberto Cingolani, and Dario Pisignano*
}

NNL, National Nanotechnology Laboratory of CNR-INFM, Università degli Studi del Salento c/o Distretto Tecnologico ISUFI, via Arnesano, I-73100 Lecce, Italy

We investigate the rheological properties of a non-Newtonian glass-former liquid within lithographically defined microchannels in the range of temperatures above the vitrification region. The non-Newtonian behavior of the fluid, as evidenced by rotational rheology, is well described by a power law dependence of the viscosity on the shear rate. Taking into account such non-Newtonian character in the equations for the microfluidic motion, we relate the penetration dynamics into capillaries with the liquid rheological properties. The temperature dependence of the viscosity, determined over 1 order of magnitude in the temperature range $286-333 \mathrm{~K}$ and for shear rates between 0.07 and $1 \mathrm{~s}^{-1}$, can be described by a Vogel-Fulcher-Tamman law, consistent with the fragile nature of the investigated compound. Microfluidics is a promising analytical approach for the investigation of the rheology of non-Newtonian fluids within confined microenvironments.

In the past decade, new miniaturization technologies have been implemented and developed to increase the throughput and to reduce the cost for the realization of microstructures, in its turn determining rapid progress in microfluidic and microelectromechanical systems. In particular, microfluidic applications ${ }^{1}$ need microstructures to define channels and to enhance mixing of liquids, heat, and mass transfer, and ultimately reactions in chemical processes. In addition, the precise control of the microfluid flow in components such as micropumps and valves, ${ }^{2,3}$ and in devices such as diagnostic lab-on-chips ${ }^{4}$ and intravenous drug delivery systems, ${ }^{5,6}$ also require a fundamental understanding of the behavior and of the rheological and structural properties of liquids within microchannel environments.

However, the fundamentals of transport phenomena and rheology in such systems are still unclear, thus making difficult the quantitative prediction of the device performances. This is especially true for many fluids of remarkable biochemical interest,

\footnotetext{
* To whom correspondence should be addressed. E-mail: dario.pisignano@ unile.it.

(1) Squires, T. M.; Quake, S. R. Rev. Mod. Phys. 2005, 77, 977.

(2) Song, Y. J.; Zhao, T. S. J. Micromech. Microeng. 2001, 11, 713-719.

(3) Unger, M. A.; Chou, H.; Thorsen, T.; Sherer, A.; Quake, S. R. Science 2000, $288,113$.

(4) Harrison, J. D.; Fluri, K.; Seller, K.; Fan, Z. H.; Effenhauser, C. S.; Manz, A. Science 1993, 261, 895-897.

(5) Blackshear, P. J. Sci. Am. 1979, 241, 52-59.

(6) Penn, R. D.; Paice, J. A.; Gottschalk, W.; Ivankovich, A. D. J. Neurosurg. 1984, 61, 302-306.

such as blood ${ }^{7}$ and organic compounds, which exhibit a complex behavior because of their nonlinear mechanical properties and peculiar viscoelasticity. ${ }^{8}$ The classical Lucas-Washburn ${ }^{9}$ equation is often not able to describe the dynamics of fluids within microchannels. In particular, since the penetration motion in microscale capillaries is strictly related to the viscosity of the filling liquid, its investigation can provide a tool for determining the rheological properties and the dynamics of the glass transition occurring in organic fluids upon decreasing temperature.

The most direct evidence of glass transition connected with a temperature reduction is the continuous yet dramatic increase of the liquid viscosity $(\eta)$ and of the structural relaxation time. The slowing down of the structural dynamics can be described by the Vogel-Fulcher-Tamman (VFT) law: ${ }^{10}$

$$
\eta(T)=\eta_{0} \exp \frac{D T_{0}}{T-T_{0}}
$$

where $\eta_{0}$ indicates the viscosity at very high temperatures, $D$ is the so-called strength parameter (fragile liquids exhibit values of $D<12$ ), accounting for the departure of the structural dynamics from an Arrhenius behavior, and $T_{0}$ (Vogel temperature) is the temperature for which a divergence of the viscosity and of the structural relaxation time is predicted. $T_{0}$ lies below the glass transition temperature, $T_{\mathrm{g}},{ }^{11}$ thus being dynamically inaccessible. In general, the variation of viscosity versus temperature makes glass-formers very suitable candidates to be studied by the observation of microfluidic motion, whose rate of penetration into microchannels increases upon decreasing the fluid viscosity. In addition, fragile fluids are particularly promising for temperatureresolved microfluidic measurements, since showing large changes in their dynamical properties upon approaching the glass transition, due to the great number of structural configurations allowed to the molecular system.

Furthermore, much evidence indicated the existence of a temperature region, well above the glass transition, where additional phenomena occur in glass-forming fluids, including changes in the temperature dependence of the structural relax-

(7) Chakraborty, S. Lab Chip 2005, 5, 421.

(8) Groisman, A.; Steinberg, V. Nature 2000, 53, 405.

(9) Washburn, E. W. Phys. Rev. 1921, 17, 273.

(10) Vogel, H. Phys Z. 1921, 22, 645. Fulcher, G. S. J. Am. Ceram. Soc. 1925, 8, 339. Tamman, G.; Hesse, W. Anorg. Allg. Chem. 1926, 156, 245.

(11) The definition of $T_{g}$ is usually done on kinetic bases. For instance, in viscoelasticity measurements, one considers the temperature at which the viscosity reaches a value of $10^{13} \mathrm{P}\left(1 \mathrm{P}=10^{-1} \mathrm{~Pa} \cdot \mathrm{s}\right)$. 


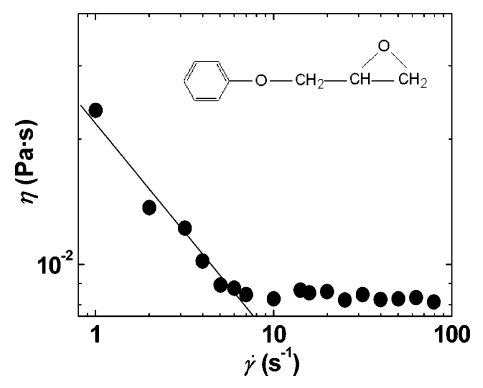

Figure 1. PGE viscosity dependence on the shear-rate, measured by classical rheometry at room temperature $(T=290 \mathrm{~K})$. The line is a fit by eq $4 \mathrm{a}$ for $\dot{\gamma}<8 \mathrm{~s}^{-1}$. Inset: molecular structure of PGE.

ation dynamics, ${ }^{12}$ frequency decoupling between main and secondary relaxation processes, ${ }^{13}$ increase of the intermolecular cooperativity, ${ }^{14}$ and departure from Debye-Stokes-Einstein relations between rotational and translational degrees of freedom. ${ }^{15}$ In particular, it was found that a unique VFT law is often not able to describe the experimental data both below and above such a crossover temperature region. A derivative analysis was proposed by Stickel et al. to evidence the changes of dynamics, consisting in linearizing the VFT law by plotting the following quantity, $\Phi: 12$

$$
\Phi=\{-\mathrm{d}[\log (\eta)] / \mathrm{d} T\}^{-1 / 2}
$$

Microfluidics permits us to investigate such a strategic range of temperatures operating with very small volumes of sample (in the $\mathrm{pL}-\mu \mathrm{L}$ range) under conditions of laminar flows and high surface to volume ratio.

In this work, we focus on the investigation of the flow behavior in microchannels as an analytical route to determine the rheological properties of non-Newtonian complex fluids and the dependence of their functional parameters on the shear rate $(\dot{\gamma})$ within the microchannels. We study a prototype non-Newtonian glass-former liquid, phenyl glycidyl ether (PGE), by the direct observation of the penetration dynamics into microfabricated channels and a model integrating the Navier-Stokes description of the flow dynamics with a power law equation accounting for the non-Newtonian properties of the fluid. The variations of $\eta$ are studied over 1 order of magnitude in the temperature range 286$333 \mathrm{~K}$ and for $\dot{\gamma}$ between 0.07 and $1 \mathrm{~s}^{-1}$. This approach can open new perspectives for both the basic understanding of the structural dynamics of complex fluids within microenvironments and the optimization of the flow operational conditions of biodiagnostic and analytical lab-on-chips.

\section{EXPERIMENTAL SECTION}

The epoxy monomer PGE, with molecular weight of $\sim 150$ (molecular structure displayed in the inset of Figure 1) and boiling point of $\sim 520 \mathrm{~K}$, was purchased from Fluka (Milan, Italy). The compound, which is in liquid form at room temperature, was used as received, without dilution in any solvent. Rheological measurements were first carried out by a rotational rheometer (TA

(12) Stickel, F.; Fischer, E. W.; Richert, R. J. Chem. Phys. 1995, 102, 6251.

(13) Corezzi, S.; Campani, E.; Rolla, P. A.; Capaccioli, S.; Fioretto, D. J. Chem. Phys. 1999, 111, 9343

(14) Casalini, R. K. L.; Ngai, K. L.; Roland, C. M. C. M. Phys. Rev. B 2003, 68, 014201.

(15) Rössler, E. Phys. Rev. Lett. 1990, 65, 1595.
Instruments Inc., New Castle, DE) equipped with a dual-range rebalance transducer. A couette geometry was employed, with bob and cup size of 34 and $32 \mathrm{~mm}$, respectively, and with a sample volume of $15 \mathrm{~mL}$ and applied shear rates between 1 and $10^{2} \mathrm{~s}^{-1}$.

Master structures employed for templating the poly(dimethylsiloxane) (PDMS) elements were fabricated by photolithography. After the deposition of standard photoresist (Clariant AZ5214E: AZ EBR Solvent 1:1), UV exposure (8'30" at $45 \mathrm{~W}$ ) and development, we etched the thermal dioxide by using $\mathrm{NH}_{4} \mathrm{~F} / \mathrm{HF} /$ $\mathrm{H}_{2} \mathrm{O}(6.6 \mathrm{~g} / 1.6 \mathrm{~mL} / 10 \mathrm{~mL})$. The so-obtained $\mathrm{SiO}_{2}$ mask was then employed to etch silicon by use of $5.9 \mathrm{M}$ solutions of $\mathrm{KOH}$ at $80^{\circ} \mathrm{C}$. Monocrystalline (100) silicon samples were etched along $\{111\}$ walls inclined at $54^{\circ}$ with respect to the bottom of the microchannels. The elastomeric replicas were then obtained by in situ polymerization $\left(140^{\circ} \mathrm{C}, 1^{\prime}\right)$ of PDMS (Sylgard 184 by Dow Corning, Midland, MI, A/B 1:9).

The microfluidic device was featured by parallel microchannels, of depth $(h) \cong 1.2 \mu \mathrm{m}$, width $(W) \cong 17.0 \mu \mathrm{m}$, and length $(L) \cong 13$ $\mathrm{mm}$, and it was realized by the conformal contact superposition of a textured PDMS element, negatively replicating the $\mathrm{Si}-\mathrm{SiO}_{2}$ master template and a substrate of $\mathrm{Si}-\mathrm{SiO}_{2}$ (oxide thickness $\cong$ $100 \mathrm{~nm}$ ). The employed microchannels exhibit therefore roughly trapezoidal cross sections, with three walls made by PDMS and the bottom surface made by $\mathrm{SiO}_{2}$. The overall balance of surface tensions favors the spontaneous penetration of PGE in the microchannels, in agreement with previous reports on microfluidic filling by similar systems. . $^{16,17}$

To study the microfluidic penetration dynamics, we released $5 \mu \mathrm{L}$ of PGE from a Gilson pipet at the edge of the microchannels, which were then spontaneously filled by capillary action. The filling rate during the capillary rise was observed by optical microscopy through a lateral ruler integrated in the chip, having parallel ticks separated by $70 \mu \mathrm{m}$. Homemade software allowed us to determine the exact position of the fluid front along the microchannels during the entire filling process. The system temperature was varied between 286 and $333 \mathrm{~K}$ by means of a Peltier element (Melcor, Trenton, NJ) having an area of $12 \times 12 \mathrm{~mm}^{2}$ and supporting a maximum current of $2.1 \mathrm{~A}$ and a maximum power of $4.5 \mathrm{~W}$. The Peltier element was placed directly in contact with the rear side of the substrate, and the temperature in the microfluidic channels was measured by a Pt100 sensor, connected to the bottom surface of the capillaries. The system temperature was calibrated and stabilized within $\pm 0.1 \mathrm{~K}$ by an electronic proportional integral derivative controller ( $\gamma 2$ series by Ascon, Milan, Italy), supplying current to the Peltier element.

With our capillary geometry, we observed that temperatures lower than $286 \mathrm{~K}$ led to a drop of the filling rate of the fluid into microchannels, whereas temperatures higher than $333 \mathrm{~K}$ could cause a loss of mutual adhesion between the chip surfaces, probably favored by the penetration of liquid between adjacent channels. We could not observe significant thermal gradient effects and any evaporation phenomena during measurements.

\section{MODEL}

The flow of a liquid inside a capillary with longitudinal coordinate, $z$, is generally described by the following Poiseuille

(16) Kim, E.; Xia, Y.; Whitesides, G. M. J. Am. Chem. Soc. 1996, 118, 5722.

(17) Pisignano, D.; Sariconi, E.; Mazzeo, M.; Gigli, G.; Cingolani, R. Adv. Mater. 2002, 14, 1565. 
law, which is obtained by the solution of the Navier-Stokes equation under conditions of a preponderance of viscous forces over inertial forces ${ }^{1}$ and laminar flow for Newtonian fluids. These classes of fluids present a linear relation between the shear stress $(\tau)$ and the shear rate $(\tau=\eta \dot{\gamma})$, with a viscosity not depending on shear. They are described by

$$
\mathrm{d} P / \mathrm{d} z=\eta \nabla^{2} u_{z}
$$

where $u_{z}$ indicates the fluid velocity in microchannels, the right member of eq 3 thus being related to the viscous force acting on the liquid, and $P$ is the total effective pressure forcing the liquid along the microchannel. ${ }^{18}$ The previous expression provides, $\mathrm{d} V /$ $\mathrm{d} t=\pi R^{4} / 8 \eta \mathrm{d} P / \mathrm{d} z$, where $\mathrm{d} V$ is the volume of the liquid flowing through any cross section of the capillary in the time, $\mathrm{d} t$, and $R$ is hydraulic radius of the capillary. In the case of additional slipping effects, the previous equation becomes, $\mathrm{d} V / \mathrm{d} t=\pi / 8 \eta \mathrm{d} P / \mathrm{d} z\left(R^{4}\right.$ $+4 \epsilon R^{3}$ ), where $\epsilon$ is the coefficient of slip of the liquid. ${ }^{9}$ In general, the driving pressure is composed of three terms: the unbalanced atmospheric pressure, the hydrostatic pressure, and the capillarity pressure $\left(P_{\mathrm{c}}\right)$ expressed by the Young-Laplace equation, namely, $P_{\mathrm{c}}=2 \sigma(\cos \theta) / R$, where $\sigma$ and $\theta$ indicate the surface tension of the liquid and its effective contact angle with respect to the channel walls, respectively. ${ }^{19,20}$ In horizontal capillaries, neglecting wall slip effects and external pressure, the Poiseuille equation becomes, $\mathrm{d} z / \mathrm{d} t=(R \sigma \cos \theta) / 2 \eta z$, where $z$ is the length of the column of liquid inside the capillary at time $t$. Integrating gives the LucasWashburn equation for the penetration dynamics, which is usually employed to describe the advancement motion of a fluid front in the capillary: ${ }^{16,21,22} z=B t^{\beta}$, where $\beta=0.5$ and $B=\left(R^{2} P_{\mathrm{c}} / 4 \eta\right)^{1 / 2}{ }^{23}$ In the previous expression, the temperature dependence of $B$ is mainly determined by the fluid viscosity. We note that experimental findings for organics in microchannels often exhibit different behaviors, characterized by a capillary flow more effectively halted, upon increasing time, than a $t^{0.5}$ law. ${ }^{21}$

In addition, an important issue for non-Newtonian fluids is the dependence of the viscosity on the shear rate, $\dot{\gamma}$. In particular,

(18) The generalied form of eq 3 , also taking into account compressible fluids, is: $\rho D \mathbf{u} / D t=-\nabla P+\eta \nabla \cdot\left[\nabla \mathbf{u}+(\nabla \mathbf{u})^{+}\right]$, where $D / D t=\partial / \partial t+\mathbf{u} \cdot \nabla$ and $(\nabla \mathbf{u})^{+}$indicates the transpose of $\nabla \mathbf{u}$. See ref 25 . The treatment at the basis of both the classical Lucas-Washburn equation for capillary dynamics and our model relies then on the condition of viscous force density much larger than inertial force density. See ref 1 . There is wide evidence for the validity of this modelling approach for describing many fluid systems, such as poly(ethylene) oxides (viscosities of the order $10^{-3}-10^{-2} \mathrm{~Pa} \cdot \mathrm{s}$ ), hydrolyzed polyacrylamide (viscosities of the order $10^{-2}-10^{-1} \mathrm{~Pa} \cdot \mathrm{s}$ ) and polyurethanes (viscosities in the range of $10^{-1} \mathrm{~Pa} \cdot \mathrm{s}$ ). See refs 17 and 27.

(19) For our microchannel geometry, embedding one (hydrophilic) $\mathrm{SiO}_{2}$ and three (hydrophobic) PDMS surfaces, the wettability from PGE is different along the different capillary walls (with liquid-surface contact angles $\theta_{\text {PDMS }}$ and $\theta_{\mathrm{SiO}_{2}}$, respectively), see the inset of Figure $2 \mathrm{~b}$. The corresponding form of the capillarity pressure is $P_{\mathrm{c}} \cong\left(\sigma\left[(2 h+W) \cos \theta_{\mathrm{PDMS}}+W \cos \theta_{\mathrm{SiO}_{2}}\right]\right) /$ $W h$, which can be brought to the Young-Laplace form by defining the effective contact angle by, $\cos \theta=(2 h / W+1) \cos \theta_{\mathrm{PDMS}}+\cos \theta_{\mathrm{SiO}_{2}}$ and the hydraulic radius as $R=2 h$.

(20) Delamarche, E.; Bernard, A.; Schmid, H.; Bietsch, A.; Michel, B.; Biebuyck, H. J. Am. Chem. Soc. 1998, 120, 500.

(21) Jeon, N. L.; Choi, I. S.; Xu, B.; Whitesides, G. M. Adv. Mater. 1999, 11, 946.

(22) Pisignano, D.; Di Benedetto, F.; Persano, L.; Mele, E.; Cingolani, R. Synth. Met. 2005, 153, 325.

(23) Martic, G.; De Coninck, J.; Blake, T. D. J. Colloid Interface Sci. 2003, 263, 213. many non-Newtonian fluids can be described by a power law model by Ostwald and de Waele: ${ }^{24}$

$$
\begin{gathered}
\eta=m \dot{\gamma}^{n-1} \\
\tau=m \dot{\gamma}^{n}
\end{gathered}
$$

where $m\left(\mathrm{~Pa} \cdot \mathrm{s}^{n}\right)$ is the flow consistency coefficient and $n$ (dimensionless) is a flow behavior index, depending on the investigated fluid.

A Newtonian representation is recovered with $n=1$ and $m=$ $\eta$. If $n<1$, the fluid is said to be pseudoplastic (shear thinning), whereas if $n>1$, the fluid is dilatant (i.e., shear thickening). Employing eqs $4^{25,26}$ in the simplified case of a cylindrical capillary leads one to the following equation, instead of eq 3 :

$$
\frac{\mathrm{d} P}{\mathrm{~d} z}=\frac{1}{r} \frac{\partial}{\partial r}\left[m r\left(\frac{\mathrm{d} u_{z}}{\mathrm{~d} r}\right)^{n}\right]
$$

Again, the left member of eq 5 takes into account the capillary pressure force, and the right term is related to the viscous force. The solution of eq 5 , for no slip effect at the walls and for $n<1$, is

$$
\frac{\mathrm{d} z}{\mathrm{~d} t}=\frac{G}{(m)^{1 / n}}\left(\frac{P_{\mathrm{c}}}{z}\right)^{1 / n}
$$

where $G$ depends on the geometry of the microfabricated channel and on $n$. The rate of penetration into microchannels is therefore inversely proportional to the $1 / n$ power of the flow consistency coefficient, instead of being inversely proportional to $\eta$ as in the Newtonian case, with a volumetric flow given by, $\mathrm{d} V / \mathrm{d} t=$ $\pi n R^{3 n+1 / n} /(3 n+1)(1 / 2 m \mathrm{~d} P / \mathrm{d} z)^{1 / n}$. Eventual slipping effects can be incorporated in the $G$ factor, without substantially affecting the resulting $z(t)$ functional law. The previous equation finally gives

$$
z=\tilde{\mathrm{G}}\left(\frac{P_{\mathrm{c}}}{m}\right)^{1 /(n+1)} t^{n /(n+1)}=A t^{n /(n+1)}
$$

where $\tilde{G}=((n+1) / n)^{n / n+1} G^{n /(n+1)}$ and $A$ is a phenomenological constant, depending on the system temperature mainly through the flow consistency coefficient $m$, in its turn related to the viscosity by eq $4 \mathrm{a}$. Therefore, eq 7 allows one to take into account the non-Newtonian behavior of the penetrating fluid by a more general expression of the exponent $\beta$, which is now given by the ratio $n /(n+1)$.

Furthermore, since $\dot{\gamma}$ varies along the microchannel, it is useful to obtain a relation between the capillary filled length and the shear-rate. To this aim, considering $\dot{\gamma} \approx \mathrm{d} z / \mathrm{d} t / h,{ }^{27}$ one has

(24) Ostwald, W. Kolloid-Z. 1925, 36, 99. De Waele, A. Oil Color Chem. Assoc. J. 1923, 6, 33.

(25) Bird, R. B.; Curtiss, C. F.; Armstrong, R. C. Dynamic of Polymeric Liquids; Wiley: New York, 1960.

(26) Tritton, D. J. Physical Fluid Dynamics; Clarendon: Oxford, 1987.

(27) Srivastava, N.; Burns, M. A. Anal. Chem. 2006, 78, 1690. 


$$
z=\frac{G^{n} P_{\mathrm{c}}}{h^{n} m} \cdot \frac{1}{\dot{\gamma}^{n}}
$$

Consequently, experimental $(z, \dot{\gamma})$ curves collected at different temperatures can be related to the temperature dependence of the flow consistency coefficient and hence of the system viscosity.

\section{RESULTS AND DISCUSSION}

We first investigated our monoepoxy with classical rheometry as reference. The resulting PGE viscosity dependence on the shear rate is displayed in Figure 1, clearly indicating a roughly Newtonian and a non-Newtonian behavior for $\dot{\gamma}>10 \mathrm{~s}^{-1}$ and for $\dot{\gamma}<10 \mathrm{~s}^{-1}$, respectively. At high shear rate values, the viscosity is almost constant around $8 \times 10^{-3} \mathrm{~Pa} \cdot \mathrm{s}$. For low $\dot{\gamma}$, the system can be effectively described by the Ostwald and de Waele laws, eq $4 \mathrm{a}$, with flow behavior index $n=0.48 \pm 0.04$.

The microfluidic $(z, t)$ and $(z, t)$ data were then collected by realtime observation, and the penetration curves were fitted by eq 7 (Figure 2). The liquid-solid interfacial tensions in the microchannels favor the spontaneous penetration of PGE (contact angle photographs in the inset of Figure 2b). The microfluidic data of PGE are well described by eq 7 , with an exponent $\beta=n /(n+1)$ $=0.29 \pm 0.02$, corresponding to $n=0.41 \pm 0.05$ (Figure 2a). Within experimental errors, this is in agreement with the value of the flow behavior index obtained by classical rheometry. The liquid fills completely the microchannels (having length, $L=13$ $\mathrm{mm}$ ) in $5400 \mathrm{~s}$ at $333 \mathrm{~K}$, and the residual velocity of the fluid front at the end of capillary is $0.7 \mu \mathrm{m} / \mathrm{s}$. At $286 \mathrm{~K}$, the fluid penetrates into the channels up to a maximum value, $z=2.5 \mathrm{~mm}$, in a time as large as $5400 \mathrm{~s}$. At the end of the filling process, the penetration velocity is as low as $0.1 \mu \mathrm{m} / \mathrm{s}$. Therefore, decreasing the temperature by $\sim 50 \mathrm{~K}$ causes a residual penetration speed almost seven times lower at the end of the experimental observation. Such a remarkable decrease is connected to the glass transition priming of the fragile monoepoxy, which directly affects the capillary motion because of the abrupt increase of the fluid viscosity upon decreasing temperature. Stronger fluids than PGE would lead to a less evident reduction of the microfluidic penetration rates upon decreasing temperature.

We then applied the non-Newtonian modeling of the capillary penetration to extract the variation of the system viscosity upon decreasing temperature and the functional parameters describing the structural dynamics of PGE. In the following, the weak dependence of $n$, of the contact angle, $\theta$, and of the surface tension, $\sigma$, on the temperature ${ }^{25,28}$ will be neglected with respect to exponential-like (VFT) behaviors, such as that of $\eta$. We first plotted the $(z, \dot{\gamma})$ curves (Figure 3 ), which are generally well described by a $z \sim \dot{\gamma}^{-n}$ law such as that of eq 8 , as shown in the

(28) De Ruijter, M.; Kölsch, P.; Voué, M.; De Coninck, J.; Rabe, J. P. Colloids Surf. A 1998, 144, 235.

(29) In Figure 3, some kinks in the data collected at different system temperatures are appreciable, due to the roughness of the $(z, \dot{\gamma})$ curves. In previous studies, similar effects were attributed to local disuniformities of the liquid/ wall interfacial energies - see ref 22 . These could be induced by minor amounts of organic contaminants or local surface roughness due to the lithographic processing and etching. However, as observable by the fitting curves in Figure 2a and in the inset of Figure 3, we did not found any appreciable influence of these effects on the overall microfluidic penetration dynamics.

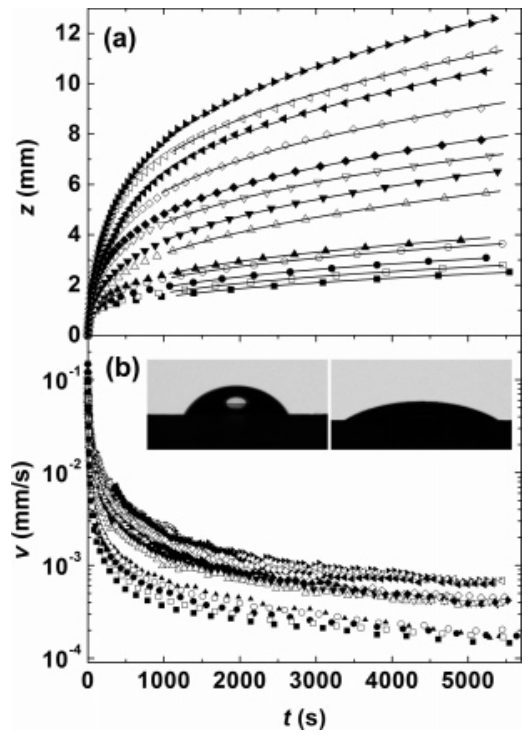

Figure 2. (a): Covered length, $z$, vs time, $t$, at different temperatures, with the best fits according to eq 7. (b): Filling rate, $z$, vs time, $t$, at different temperatures., $286 \mathrm{~K} ; \square, 288 \mathrm{~K} ; \bullet, 290 \mathrm{~K} ; \bigcirc, 292 \mathrm{~K}$; $\Delta, 295 \mathrm{~K} ; \Delta, 298 \mathrm{~K} ; \mathbf{\nabla}, 303 \mathrm{~K} ; \nabla, 308 \mathrm{~K} ; \diamond, 313 \mathrm{~K} ; \diamond, 318 \mathrm{~K}$; leftfacing solid triangle, $323 \mathrm{~K}$; left-facing open triangle, $328 \mathrm{~K}$; rightfacing solid triangle, $333 \mathrm{~K}$. Inset of $(\mathrm{b})$ : Photographs of the contact angle, $\theta$, for $3-\mu \mathrm{L}$ drops of PGE on a PDMS surface (left inset, $\theta=$ $61^{\circ}$ ) and on a $\mathrm{SiO}_{2}$ surface (right inset, $\theta=31^{\circ}$ ).

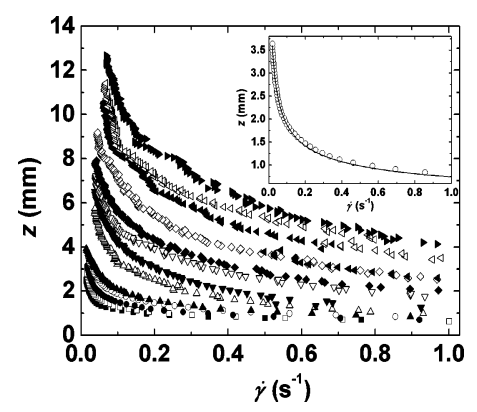

Figure 3. Covered length, $z$, vs shear rate $\left(\dot{\gamma}\right.$ between $7 \times 10^{-2}$ and $\left.9 \times 10^{-1} \mathrm{~s}^{-1}\right)$ at different temperatures. Same symbols as those used in Figure 2. Inset: $(z, \dot{\gamma})$ curve for $T=292 \mathrm{~K}$ (empty circles), and corresponding best fit to eq 8 (superimposed line).

inset of Figure $3 .{ }^{29}$ Then, in order to extract the temperature dependence of the PGE viscosity from the measured $(z, \dot{\gamma})$ curves, we determined the ratios between the coordinate filled at room temperature, $T_{\mathrm{RT}}$, and those at each investigated temperature, $T_{i}$, for a fixed value of $\dot{\gamma}: z\left(\dot{\gamma}, T_{\mathrm{RT}}\right) / z\left(\dot{\gamma}, T_{i}\right)$. This directly provides the quantity, $\eta\left(\dot{\gamma}, T_{i}\right) / \eta\left(\dot{\gamma}, T_{\mathrm{RT}}\right)$, by taking into account eqs $4 \mathrm{a}$ and 8 (Figure 4a). The viscosity curves were then normalized by employing the value of the room-temperature viscosity for $\dot{\gamma}=1$ $\mathrm{s}^{-1}$, which was measured to be $2.3 \times 10^{-2} \mathrm{~Pa} \cdot \mathrm{s}$ (see Figure 1 ). This procedure allowed us to obtain the $\eta(\dot{\gamma})$ curves of PGE within the microfluidic device down to $0.07 \mathrm{~s}^{-1}$, for different values of temperature, as shown in Figure 4b. We underline that classical rheometry hardly allows one to explore, for low-viscosity, glassforming systems, such a range of very low shear rates that is

(30) This limitation of classical rheometry is due to the poor values of the torques (of the order of $10^{-2} \mathrm{~g} \cdot \mathrm{cm}$ or less) that can be associated with shear rates below $1 \mathrm{~s}^{-1}$ for system viscosities of the order of $10^{-1} \mathrm{~Pa} \cdot \mathrm{s}$ or less, such as the viscosity of PGE and of a wide range of other glass-forming systems. 


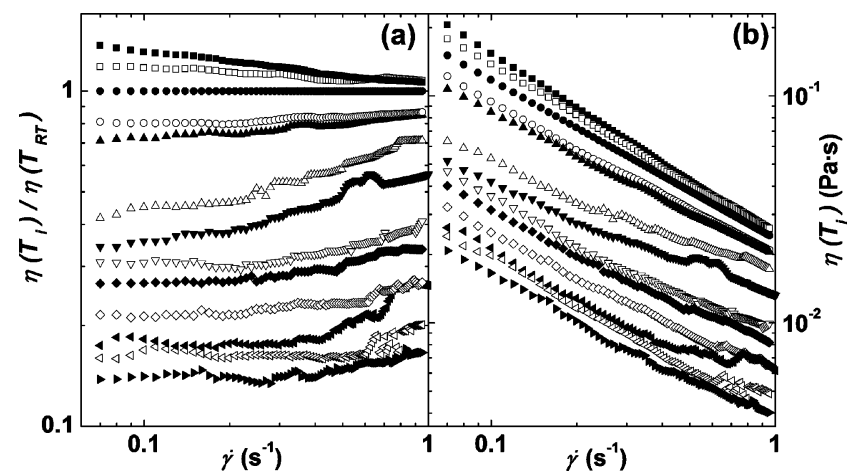

Figure 4. (a) Ratios between viscosities at different temperatures, $T_{i}$, vs shear rate; (b) viscosity vs shear rate at different temperatures. Same symbols as those used in Figure 2.

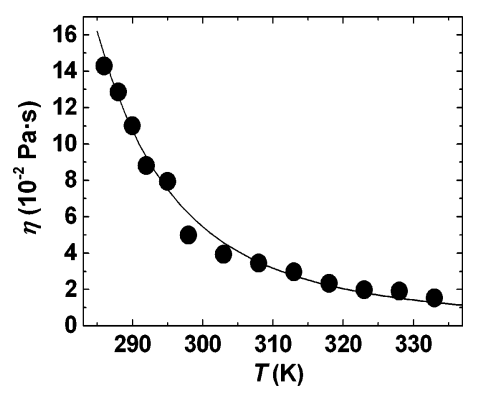

Figure 5. Temperature dependence of the viscosity of PGE in microchannels for $\dot{\gamma}=0.1 \mathrm{~s}^{-1}$ (full circles). Superimposed line: corresponding VFT fit curve.

instead accessible to microfluidics (and that is critical for the microfluidic behavior of fluids). ${ }^{30}$ We found that, at each temperature, the PGE microfluidic viscosity increases upon decreasing $\dot{\gamma}$ according to a power law. For instance, at $333 \mathrm{~K}$ we found a variation of $\eta$ from $4.0 \times 10^{-3}$ (for $\dot{\gamma}=1 \mathrm{~s}^{-1}$ ) to $2.1 \times 10^{-2} \mathrm{~Pa} \cdot \mathrm{s}$ (for $\dot{\gamma}=0.07 \mathrm{~s}^{-1}$ ).

In addition, for each value of the shear rate, the viscosity decreases upon increasing the system temperature, for instance, varying from $1.4 \times 10^{-1}$ (at $T=286 \mathrm{~K}$ ) to $1.5 \times 10^{-2} \mathrm{~Pa} \cdot \mathrm{s}$ (at $T=$ $333 \mathrm{~K})$ for $\dot{\gamma}=10^{-1} \mathrm{~s}^{-1} \cdot \mathrm{d} \eta / \mathrm{d} T$ is found to be large for low temperatures, progressively decreasing when the temperature increases. This behavior is well described by a unique VFT law (Figure 5). To investigate this issue in depth, we performed the Stickel derivative analysis on the extracted viscosity data, finding that no significant changes are observable in the structural dynamics within the investigated temperature range, as shown in Figure 6.

The strength parameter, $D$, and the Vogel temperature, $T_{0}$, are comprised in the range $1-5$ and $170-215 \mathrm{~K}$, respectively, confirming the fragile nature of the investigated molecular system. The resulting glass transition temperature, given by the VFT law with a viscosity value of $10^{12} \mathrm{~Pa} \cdot \mathrm{s}$, is $T_{\mathrm{g}}=T_{0}\left\{1+D / \ln \left[10^{12} /\right.\right.$ $\left.\left.\eta_{0}(\mathrm{~Pa} \cdot \mathrm{s})\right]\right\}$. For the data of Figure 5 , this provides $T_{\mathrm{g}} \cong 238 \mathrm{~K}$. For sake of comparison, we recall that the previously reported value of $T_{\mathrm{g}}$ for the PGE compound is $\sim 200 \mathrm{~K}$, obtained by low-temperature dielectric spectroscopy measurements. ${ }^{31}$ Such a discrepancy is physically reasonable, given the dynamic changes often observed in epoxy glass-formers at the crossover temperature region. ${ }^{13}$ In addition, concerning the dependence of

(31) Justl, A. Thesis Degree, University of Bayreuth, 2000.

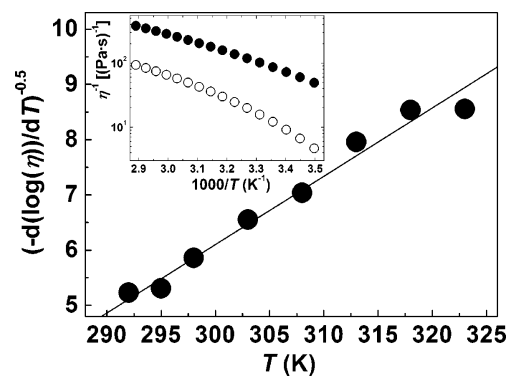

Figure 6. Stickel plot of viscosity data versus temperature (full circles), with linear curve fit (solid line) for $\dot{\gamma}=0.1 \mathrm{~s}^{-1}$. Inset: Arrhenius plot depicting the temperature dependence of the viscosity for $\dot{\gamma}=$ $0.07 \mathrm{~s}^{-1}$ (open circles) and for $\dot{\gamma}=1 \mathrm{~s}^{-1}$ (full circles), respectively.

the temperature behavior of the structural dynamics on the shear rate, the slope and the curvature of the viscosity data indicate a slightly stronger behavior of the system for larger values of $\dot{\gamma}$ (inset of Figure 6). In terms of the energy landscape of glass-formers, ${ }^{32}$ namely, the $3 N+1$-dimensional hypersurface representation of the fluid potential energy as a function of the $N$ particles coordinates, the structural dynamics slows down upon decreasing temperature because of the reduced capability of the system to explore different configurational states. Different factors may block the exploration of many configurations by the fluid, both thermodynamic and kinetic: a "thermodynamically strong" system is characterized by spaced out potential energy minimums, a "kinetically strong" one has energy minimums that, although possibly denser, are separated by high barriers and are therefore hardly reachable by the system at a given temperature. ${ }^{33}$ In fact, the energy landscape is not a fully intrinsic feature of a molecular systems, being instead sensitive to many parameters including the liquid density, pressure, etc. The behavior observed in our experiments suggests an impact of the fluid motion in microchannels on the energy landscape determining the material fragility. In particular, the deformation-induced dilatation is expected to provide an amount of free volume available, or in other words, higher values of the shear stress could determine more instability in the molecular system, thus increasing its thermodynamic fragility. The rheology of metallic glasses has been recently analyzed through a cooperative shear flow model accounting for such barrier softening. ${ }^{34}$ Further studies are needed to assess whether a kinetic fragility could be introduced in the confined microenvironment because of the liquid-solid walls interfacial interactions.

\section{CONCLUSION}

Lithographically defined microfluidics offer unique possibilities for studying complex fluids and their temperature and glass transition dynamics in microconfined systems. Here, we propose microfluidics as an analytical approach for the investigation of the rheology of non-Newtonian fluids within microenvironments. We investigated the glass transition behavior of

(32) Angell, C. A.; Ngai, K. L.; McKenna, G. B.; McMillan, P. F.; Martin, S. W. J. Appl. Phys. 2000, 88, 3113-3157; and references therein.

(33) Angell, C. A. Proc. Natl. Acad. Sci. U.S.A. 1995, 92, 6675-6682.

(34) Demetriou, M. D.; Harmon, J. S.; Tao, M.; Duan, G.; Samwer, K.; Johnson, W. L. Phys. Rev. Lett. 2006, 97, 065502. 
the non-Newtonian fluid PGE within a microfluidic device, describing the raising of the fluid in the microchannels by a power law model. For each value of $\dot{\gamma}$, the temperature dependence of the viscosity could be described by a unique VFT law, as expected in the investigated temperature range, with strength parameters consistent with the fragile behavior of PGE. Cooled microfluidic devices with suitable geometry will also enable the investigation of the dynamic behavior for temperatures in the crossover region,where the fluid motion in the microchannels becomes very slow.

\section{ACKNOWLEDGMENT}

This work was supported by the Italian Institute of Technology and by the Italian Minister of Education, University and Research through the FIRB project "Laboratorio nazionale sulle nanotecnologie per la genomica e postgenomica" (NG-Lab).

Received for review December 20, 2006. Accepted May 21, 2007.

AC062405T 